\title{
Capturing a Concave Polygon with Two Disc-Shaped Fingers
}

\author{
Attawith Sudsang and Thanaphon Luewirawong \\ Department of Computer Engineering, \\ Chulalongkorn University, Bangkok 10330, Thailand \\ \{attawith,luewirawong\}@cp.eng.chula.ac.th
}

\begin{abstract}
A successful grasp of an object can be guaranteed when the object can never escape from the surrounding fingers during the entire grasping execution. Ability to capture an object clearly contributes to the robustness and success of grasping tasks. Object concavity is a useful geometric property allowing objects to be captured with only few fingers. In particular, certain concave objects may be captured using two fingers by appropriately placing the fingers close to some pair of opposite concave sections. Based on this intuitive idea, we address the problem of capturing concave polygonal objects with two disc-shaped fingers. We present an approach for computing a range of distance such that the two fingers can move away from a given immobilizing grasp but still prevent the object from escaping; when within this computed range, it is guaranteed under the frictionless contact assumption that the fingers can move toward each other to bring the object to the given immobilizing grasp. The proposed approach is implemented and preliminary result is presented.
\end{abstract}

\section{INTRODUCTION}

An object is captured when it is restricted to stay within a bounded region of the workspace, i.e., there exists no trajectory to bring the object to infinity. A capturing action generally applies to a set of the object's configurations rather than a single one. It provides a means with which uncertainty in the object's configuration can be handled. Ability to capture an object clearly contributes to the robustness and success of grasping tasks as we can guarantee a successful grasp of an object when it cannot escape from the surrounding fingers during the entire grasping execution. ${ }^{1}$ A comprehensive review on grasping works can be found in [2].

Object concavity is a geometric property allowing objects to be captured with only few fingers. In particular, certain concave objects may be captured using two fingers by appropriately placing the fingers close to some pair of opposite concave sections. In fact, concavity is a necessary condition for an object to be capture-able by two fingers. Concavity can also be used for guiding manipulation; see [1] for a work that takes advantage of concavity to derive a method for orienting parts using inside-out pulling.

In this paper, we address the problem of capturing and grasping a simple polygon with concavities in the plane by two identical disc-shaped fingers under the frictionless

\footnotetext{
${ }^{1}$ In static grasp computation, it is usually assumed that the object to be grasped stays fixed but this is not always true because all contacts may not be made simultaneously causing the object to move away from the initial configuration for which the grasp is computed
}

contact assumption. It is important to note that there are two main classes of two-finger grasps: (1) squeezing grasps for which the two fingers are pushing toward each other (Fig. 1(a)), and (2) stretching grasps for which the two fingers are pushing away from each other (Fig. 1(b)). Our focus is mainly on capturing issues related to squeezing grasps. Specifically, we present an approach for computing a range of distance such that the two fingers can move away from a given squeezing immobilizing grasp but still prevent the object from escaping. Moreover, when within this computed range, it is guaranteed under the frictionless contact assumption that the fingers can move toward each other to bring the object to the given immobilizing grasp. This computed range may also be used as a quality in selecting good grasps since a larger range yields better tolerance for error in positioning the fingers during the pre-grasp stage.

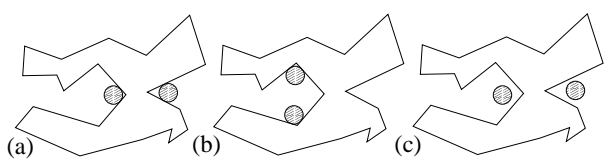

Fig. 1. An example of (a) a squeezing grasp, (b) a stretching grasp, and (c) an object being captured as the fingers move away from a squeezing grasp

Although capturing actions can help handle uncertainty in the object's configuration and appears to be useful for grasping and manipulation tasks, the problem of capturing objects has so far received little attention in robotics. It was introduced in [8] the concept of Inescapable Configuration Space (ICS) region, i.e., on the idea of characterizing the regions of configuration space in which the object is not immobilized but is constrained to lie within a bounded region of the free configuration space This concept is used in [7] as a basis for computing a plan for manipulating polygonal objects using three discshaped robots. Similar work in the two-finger case can be found in [4] where a method based on stratified Morse theory to find configurations of the gripper in the contact $\mathrm{C}$-space that can form an inescapable cage is presented. The works described above aim at solving a similar problem to ours but the approaches taken are based on analyzing regions of the dual finger/object configuration space, which results in complex computation. In contrast, our approach takes advantage of the polygonal object shape assumption and the object's concavities to derive 
a simple capturing condition that can be computed using only the distance information in the workspace of the object and the fingers. This is similar to the approach taken in [6] where the object's convexity is used in computing the width of a gap guaranteed not to let the object to pass through.

The rest of the paper is organized as follows. In Section II, we begin by characterizing all possible types of squeezing equilibrium grasps based on the location on the object's boundary at which the fingers are placed. In Section III, we show that grasps belonging only to two of the five possible types are able to immobilize the grasped object. The immobilizing grasps consist of equilibrium grasps with the two fingers at concave vertices (type-1), and equilibrium grasps with one finger on an edge and the other at a concave vertex (type-2). For each of these grasps, when the fingers slightly move away from the grasping position, the object will be able to move but still cannot escape (Fig. 1(c)). This idea is formalized in Section IV-A where we show that, for a given immobilizing grasp, there exists an associated finite interval of distance by which the fingers can be far apart from each other while still capture the object. Following its definition, this distance interval is referred to as a capturing interval. Then in Section IV-B, we propose a simple method for computing, for a given immobilizing grasp, a capturing interval such that whenever the distance between the capturing fingers is in this interval, we can also guarantee that the object will be brought to the immobilizing grasp without getting stuck anywhere else as we move the fingers toward each other (the immobilizing configuration is reached when the distance between the fingers is reduced to the minimum of the interval). Finally, we present preliminary results in Section $\mathrm{V}$ and discussion and future works in Section VI.

\section{EQUiLIBRIUM GRASPS}

A grasp of an object is modeled as a set of grasping points (fingertips) on the boundary of the object. An equilibrium grasp is a grasp whose grasping fingers can exert wrenches (not all of them are zero) through the grasping points to balance the object. A grasp achieves force-closure if the grasping fingers can exert forces and torques to cancel any external wrench. In other words, an object in a force-closure grasp can remain in equilibrium under arbitrary external disturbance and clearly, forceclosure implies an equilibrium grasp. Under the frictionless contact assumption, force-closure is a sufficient condition for immobility but it is not a necessary one. Considering the effect of curvature at the contact, it is shown in [5] that a grasp can immobilize an object without achieving force-closure.

In this section, we classify an equilibrium grasp based on the location of the fingers' centers. Note that the center of a finger must lie on a closed curve obtained from growing the object's boundary by the radius of the fingers.
Also recall that under the frictionless contact assumption, the line of force at a contact must be in the inward normal direction. In the following classification, grasps are distinguished by two features of the grown boundary, each of which indicates where the center of each finger is located. The possible features are a vertex, a straight edge and a circular arc (from growing the boundary at a concave vertex, an edge, and a convex vertex respectively). For each possible type, we describe the condition for its equilibrium.

- Type-1: (vertex,vertex). See Fig. 2(a). There are, in fact, four grasping points in this case. The center of a finger must be in the interior of the cone formed by the lines of forces at the two contact points of the other finger.

- Type-2: (vertex,straight edge). See Fig. 2(b). There are three grasping points. The corresponding three lines of forces intersect and the interior of the cone formed by the lines of forces exerted by the finger with two contact points must contain the center of the other finger. The object is immobilized by the effect of curvature of the fingers although the grasp does not achieve force-closure.

- Type-3: (straight edge, straight edge). See Fig. 2(c). The two edges must be parallel and the lines of forces from the two contact points intersect. The grasp can only achieve equilibrium but cannot immobilize the object as it is obvious that the object may slide in the direction of the edge.

- Type-4: (circular arc, straight edge). See Fig. 2(d). The finger associated with the circular arc feature must locate where the line of force at its contact is aligned with the line of force of the other finger.

- Type-5: (circular arc, vertex). See Fig. 2(e). The grasp can only achieve equilibrium. The object is not immobilized as it can rotate around the finger associated with the concave vertex.

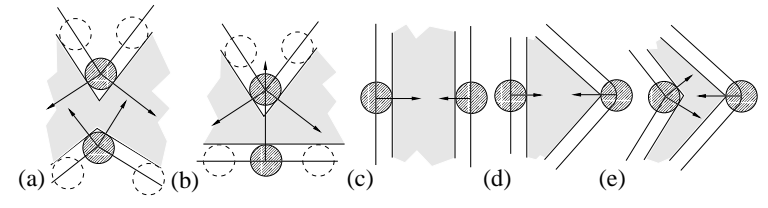

Fig. 2. Types of squeezing equilibrium grasps (see text)

As mentioned in Section I, only squeezing equilibrium grasps of types 1 and 2 can immobilize the object. This statement will be proven in the next section. As for stretching equilibrium grasps, only grasps of type- 1 can immobilize the object. This can be understood from the illustration in Fig. 3 showing that the object in a stretching grasp of type-2 may rotate around the finger at the concave vertex.

\section{IMMOBILIZING GRASPS}

In this section, we will prove that a squeezing equilibrium grasp of types 1 or 2 effectively immobilizes the 


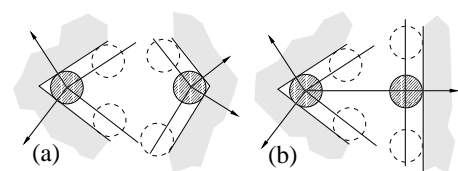

Fig. 3. Stretching equilibrium grasps of (a) type-1, and (b) type-2

grasped object. Note that there are many ways in which this statement could be proved. For example, for grasps of type 1, it is sufficient to prove that they achieve force closure and for grasps of type 2, we may use the secondorder immobility condition. A proof is recently given in [3] by considering critical values of the function of the distance between the two fingers. For completeness and to introduce elements to be used in the following sections, we will present a simple geometric proof requiring only rigidity of the grasped object and the fingers.

Let us begin with type-1 equilibrium squeezing grasp. Consider a grasp of type 1 shown in Fig. 4(a). In this illustration, we redraw Fig. 2(a) by omitting the physical boundary and showing instead the grown boundary of the object. This allows a finger to be represented as a point. From now on, without loss of generality, we consider only point fingers (only concave vertices are concerned so the grown boundaries are still polygonal). Let us denote respectively by $N$ and $M$ the top and bottom boundaries, by $N_{1}$ and $N_{2}$ the two line segments of $N$ in counterclockwise order (around the object), by $M_{1}$ and $M_{2}$ the two line segments of $M$ in counterclockwise order, and by $\boldsymbol{n}$ and $\boldsymbol{m}$ the concave vertices of $N$ and $M$ respectively. To prove that this grasp immobilizes the object, the following lemma is needed. In the lemma and the remainder of this section, we let $K$ denote the rectangular region bounded by two parallel lines $K_{m}$ and $K_{n}$ where $K_{m}$ and $K_{n}$ are the lines perpendicular to the line segment $\overline{\boldsymbol{m} \boldsymbol{n}}$ such that $K_{m}$ passes through $\boldsymbol{m}$ and $K_{n}$ passes through $\boldsymbol{n}$.

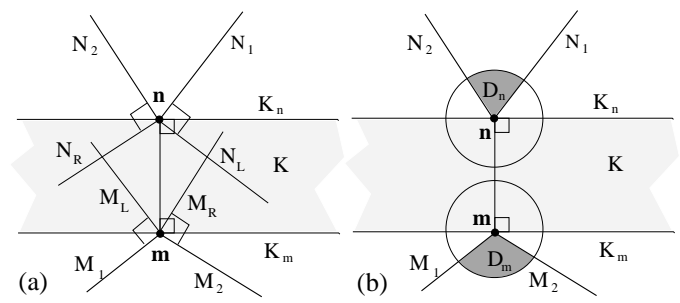

Fig. 4. A grasp of type-1 showing rectangle $K$ in between the two boundaries with (a) corresponding cones formed by the lines of forces, and (b) neighborhood of the grasping fingers

Lemma 1: The line segments $M_{1}, M_{2}, N_{1}$ and $N_{2}$ are not in $K$ except at the endpoints $\boldsymbol{m}$ and $\boldsymbol{n}$.

A proof of this lemma can be found in Appendix of this paper.

Lemma 2: An equilibrium grasp of types 1 or 2 immobilizes the grasped object.

Proof: Let us begin with type-1 where we assume without loss of generality that the object is grasped when one finger is at $\boldsymbol{m}$ and the other is at $\boldsymbol{n}$. Assume oppositely that the object can move while the two fingers stay fixed at the grasping configuration. This assumption is equivalent to assuming that the object stay fixed whereas at least one finger has to be able to move and the distance between the two fingers is fixed at $|\boldsymbol{m n}|$.

Obviously there exists a neighborhood $D_{m}$ of $\boldsymbol{m}$ that can be divided into two parts by the boundary segments $M_{1}$ and $M_{2}$ and likewise there exists a neighborhood $D_{n}$ of $\boldsymbol{n}$ that can be divided into two parts by the boundary segments $N_{1}$ and $N_{2}$ (Fig. 4(b)) . Consider the finger that is initially at $\boldsymbol{m}$ when the object is grasped. Within the neighborhood $D_{m}$, this finger can move only in the convex region bounded by $M_{1}$ and $M_{2}$ or it has to penetrate the object. Following Lemma 1, this convex region is outside the rectangle $K$ except at $\boldsymbol{m}$. Applying the same reason with the other finger, we have that if a finger moves, its new position is not in $K$. As a result, if at least one finger moves, then at least one finger is outside $K$ and the other finger is either outside $K$ or on the boundary of $K$ (at the corresponding concave vertex). Since the fingers are on different sides of $K$, the distance between them must be greater than $|\boldsymbol{m n}|$ which results in a contradiction and, therefore, completes the proof for type-1.

For type-2, using similar setup, the same argument can be applied to conclude that no matter how each finger moves (at least one finger has to be able to move) in its neighborhood, the distance between the two fingers must be greater than that when the object is grasped and that derives a contradiction which proves the lemma. In this case, one of the boundaries is a single line segment. This difference requires a straightforward modification of the idea in Lemma 1 by considering only one cone.

\section{CAPTURing OBJects}

When an object is captured by two fixed fingers, it may move but will not be able to escape from the capturing fingers. In other words, the object's configuration is restricted to lie within a bounded subset of the object's configuration space. Let us consider the positions of the fingers in a coordinate frame attached to the object being captured. In this frame, we will see that the object will stay fixed while the fingers may move within a bounded region. This allows us to view the object as a stationary obstacle and the fingers as two movable robots that are constrained to lie far apart from each other by a fixed distance. With this perspective, for a given immobilizing grasp, we will show in Section IV-A that there always exists an associated capturing interval, i.e., finite interval of distance between the two fingers for which we can guarantee that the object can be captured. Then in Section IV-B, we present a simple method for computing an associated capturing interval for a given immobilizing grasp. 


\section{A. Capturing Interval}

A capturing interval is defined as follows.

Definition 1: While still capture an object, the two fingers at a given immobilizing grasp can move away from each other with the distance between them varying within a finite interval. We call such interval of distance a capturing interval associated with the grasp.

When an object is immobilized in an immobilizing grasp, the object is certainly captured. This fact confirms that the associated capturing interval is not empty. Next, we will show that this interval is finite.

In the following discussion, let us again use a typical example of a squeezing equilibrium grasp of type 1 shown in Fig. 4(a) and all the related notations given in Section III. Let us also denote, respectively, by $\mathcal{B}_{m}$ and $\mathcal{B}_{n}$ the two fingers that lie at $\boldsymbol{m}$ and $\boldsymbol{n}$ when the object is immobilized, and pretend for a moment that the two boundaries $M$ and $N$ extend to infinity (Fig. 5(a)). We denote respectively by $M^{\prime}$ and $N^{\prime}$ the extended version of the boundaries $M$ and $N$.

Free Space Regions: Let us imagine that the fingers have moved away from each other and have the distance between them increased from $|\boldsymbol{m n}|$ to $d=|\boldsymbol{m n}|+\delta$. Now that the fingers may move (no longer at the immobilizing configuration), it is easy to see that the extended boundary $N^{\prime}$ constrains $\mathcal{B}_{n}$ to lie in the top free space portion and likewise the extended boundary $M^{\prime}$ constrains $\mathcal{B}_{m}$ to lie in the bottom free space portion. These top and bottom free space portions are denoted correspondingly by $F_{n}$ and $F_{m}$ (Fig. 5(a)).

Feasible Regions: Besides being constrained by the extended boundaries, both fingers have to maintain that they are far apart from each other by the distance $d$. Under this distance constraint, for a position to be feasible for $\mathcal{B}_{n}$, there must exist a point in $F_{m}$ that is far from the position by the distance $d$. This restriction allows us to construct the feasible region of $\mathcal{B}_{n}$ (denoted $G_{n}$ ) by growing the boundary of $F_{m}$ with the distance $d$ (Fig. 5(b)). This region does not contain $F_{m}$ for the obvious reason that $\mathcal{B}_{n}$ is forbidden to be in there by the extended boundary $M^{\prime}$. As a result, the finger $\mathcal{B}_{n}$ is now confined in both $F_{n}$ and $G_{n}$. The intersection $\hat{F}_{n}=F_{n} \cap G_{n}$ defines the region of possible positions of $B_{n}$. Following the same process, the region $\hat{F}_{m}$ of possible positions of $B_{m}$ can be obtained from $\hat{F}_{m}=F_{m} \cap G_{m}$ where $G_{m}$ is the feasible region of $\mathcal{B}_{m}$ constructed by growing boundary of $F_{n}$ with the distance $d$.

Varying $\delta$ : As mentioned earlier, the object is captured when the region of possible positions of each finger is bounded. We can see that when $\delta=0$, i.e., when the object is immobilized, $\hat{F}_{m}=\{\boldsymbol{m}\}$ and $\hat{F}_{n}=\{\boldsymbol{n}\}$ are bounded. As we increase $\delta, \hat{F}_{m}$ and $\hat{F}_{n}$ get larger in such a way that $\hat{F}_{m}$ and $\hat{F}_{n}$ for smaller $\delta$ are respectively a subset of $\hat{F}_{m}$ and $\hat{F}_{n}$ for larger $\delta$ (true from the construction of $G_{m}$ and $G_{n}$ ). This means that when the object is captured at

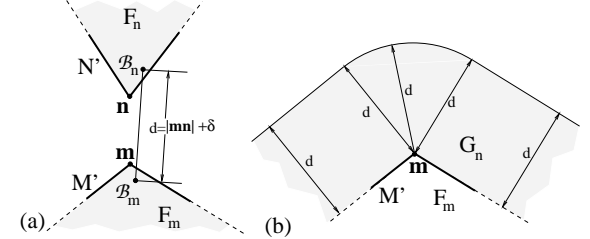

Fig. 5. The fingers are restricted to lie in (a) the free space regions by the boundary constraint, and (b) in the feasible region by the distance constraint (see text)

$\delta=\delta^{*}$, it is also captured for the interval $0 \leq \delta \leq \delta^{*}$.

Obviously, the free space regions $F_{m}$ and $F_{n}$ are fixed whereas the feasible regions $G_{n}$ and $G_{m}$ depend on $\delta$. Let us try to understand this relationship by considering the boundary of $G_{n}$ as we increase $\delta$ from zero. Fig. 6 shows snapshots of the boundary of $G_{n}$ for $\delta=\delta_{1}, \delta_{2}, \delta_{3}$ and $\delta_{4}$ where $\delta_{1}<\delta_{2}<\delta_{3}<\delta_{4}$. As we can see, as $\delta$ increases, $G_{n}$ grows larger but by the construction of feasible region and the fact that $M^{\prime}$ must be outside the rectangle $K$, subset of $G_{n}$ that is above $K_{n}$ is guaranteed to be bounded for any positive $\delta$. Combined with that the interior of the free space $F_{n}$ is above $K_{n}$, we can conclude that $\hat{F}_{n}=F_{n} \cap G_{n}$ is bounded. With the same reason, we can also conclude that $\hat{F}_{m}=F_{m} \cap G_{m}$ is bounded as well.

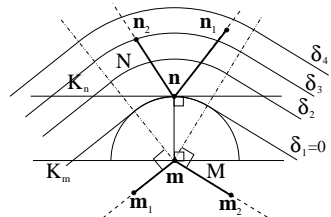

Fig. 6. The boundaries of feasible regions at varying $\delta$.

Ignoring the Boundary Extension: Now that $\hat{F}_{m}$ and $\hat{F}_{n}$ are always bounded for positive $\delta$, does it mean that the object is always captured regardless of the value of $\delta$ ? Of course, not. The "always" boundedness conclusion is due to the construction that is based on extended boundaries $M^{\prime}$ and $N^{\prime}$ (instead of the actual boundaries $M$ and $N)$. Let us try to analyze what situation the extension is not needed for ensuring the boundedness of $\hat{F}_{m}$ and $\hat{F}_{n}$. Before continuing, let $M_{x}=M^{\prime}-M$ and $N_{x}=N^{\prime}-N$ denote, respectively, the extended parts of the extended boundaries $M^{\prime}$ and $N^{\prime}$. Starting at $\delta=0$ when the object is immobilized, finger $\mathcal{B}_{n}$ can lie only at $\boldsymbol{n}$ which is on the actual boundary $N$ and finger $\mathcal{B}_{m}$ can lie only at $\boldsymbol{m}$ which is on the actual boundary $M$. Apparently, both extended parts $M_{x}$ and $N_{x}$ are not involved in constraining the fingers. Boundedness of $\hat{F}_{m}$ and $\hat{F}_{n}$ can thus be concluded in this case without the boundary extension. Let us continue by considering $\delta=\delta^{*}$ where $\delta^{*}$ is a positive constant such that the corresponding $\hat{F}_{m}$ does not contain any point of the extended part $M_{x}$ and the corresponding $F_{n}$ does not contain any point of the extended part $N_{x}$ (by continuity and finite lengths of the actual boundaries, constant $\delta^{*}$ exists; this will be explained 
in detail in Section IV-B). This means that whenever finger $\mathcal{B}_{n}$ is in contact with the object, it can be in contact only with the actual boundary $N$ (not with the extended part $N_{x}$ ). Likewise, whenever finger $\mathcal{B}_{m}$ is in contact with the object, it can be in contact only with the actual boundary $M$. In other words, the extended parts $M_{x}$ and $N_{x}$ do not participate in constraining the fingers. Therefore, we can again safely ignore the boundary extension in this case. Moreover, by the reason given in the previous paragraph, that the object is captured at $\delta=\delta^{*}$ implies that it is also captured for the entire interval $0 \leq \delta \leq \delta^{*}$. This concludes the finiteness of a capturing interval and we can then state the following lemma.
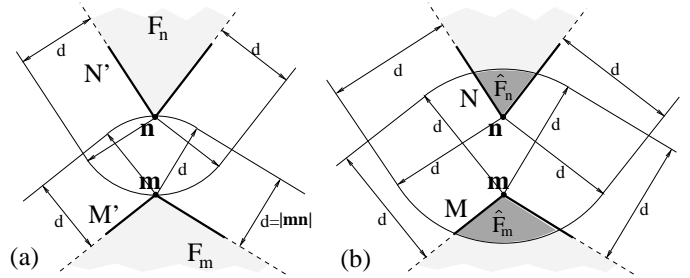

Fig. 7. The regions of possible finger positions (a) at an immobilizing grasp, and (b) as the fingers move away

Lemma 3: There exists an associated capturing interval for an equilibrium grasp of types 1 or 2 .

Although the discussion so far is written with the focus on type-1 equilibrium grasps, the line of reasoning and most arguments also apply to type-2. Another version for type- 2 can be generated by straightforwardly modifying what has been presented. Therefore, to avoid tedious repetition, it is omitted here.

\section{B. Computing a Capturing Interval}

The discussion leading to Lemma 3 has provided clues to computing a capturing interval for a given immobilizing grasp. Most relevant is the main idea that $[|\boldsymbol{m n}|,|\boldsymbol{m n n}|+$ $\left.\delta^{*}\right]$ forms a capturing interval when $\delta^{*}$ is a positive constant such that $\hat{F}_{m} \cap M_{x}=\emptyset$ and $\hat{F}_{n} \cap N_{x}=\emptyset$ when $\delta=\delta^{*}$. Such $\delta^{*}$ is claimed to exist. It turns out that computing a capturing interval is a small step from showing existence of $\delta^{*}$. Let us investigate the claim of existence by considering the boundary of the feasible region $G_{n}$ at varying values of $\delta$. Clearly, $G_{n} \cap N_{x}=\emptyset$ implies that $\hat{F}_{n} \cap N_{x}=\emptyset$ and that $G_{n} \cap N_{x}=\emptyset$ can be guaranteed when the boundary of $G_{n}$ does not intersect the extended part $N_{x}$ (if $G_{n} \cap N_{x} \neq \emptyset, N_{x}$ which is above $K_{n}$ must intersect the boundary of $G_{n}$ because it extends to infinity while the subset of $G_{n}$ above $K_{n}$ is bounded). As a consequence, it can be guaranteed that $\hat{F}_{n} \cap N_{x}=\emptyset$ when the boundary of $G_{n}$ does not intersect the extended part $N_{x}$. Now, let us trace the intersection between the boundary of $G_{n}$ and $N^{\prime}$ as we continuously increase $\delta$ from zero. At $\delta=0$, from construction, the boundary of $G_{n}$ is tangent to $K_{n}$ and intersects $N^{\prime}$ at $\boldsymbol{n}$. As $\delta$ increases, $G_{n}$ grows larger and its boundary intersects $N^{\prime}$ in two points. Since $\boldsymbol{n}$ is not an endpoint of $N$, there exists a neighborhood of $\boldsymbol{n}$ not containing any point of $N_{x}$. Therefore, by continuity, there exists a positive value of $\delta$ such that the two intersection points lie in the neighborhood which, in turn, ensures that the boundary of $G_{n}$ does not intersect $N_{x}$. Applying the same reasoning to $\hat{F}_{m} \cap M_{x}=\emptyset$, existence of $\delta^{*}$ is confirmed.

While ensuring that $\hat{F}_{m} \cap M_{x}=\emptyset$ and $\hat{F}_{n} \cap N_{x}=\emptyset$, the value of $\delta$ can be increased from zero to the point that $G_{m}$ begins to contain some points of $M_{x}$ or $G_{n}$ begins to contain some points of $N_{x}$. In other words, this critical event corresponds to the maximum $\delta$ that can ensure $\hat{F}_{m} \cap$ $M_{x}=\emptyset$ and $\hat{F}_{n} \cap N_{x}=\emptyset$. It occurs at the lowest $\delta$ such that an endpoint of $M$ lies on the boundary of $G_{m}$ or an endpoint of $N$ lies on the boundary of $G_{n}$ (will be clarified shortly). There are four endpoints to consider. Let us denote by $\boldsymbol{n}_{1}$ and $\boldsymbol{n}_{2}$ the endpoints of $N$ and by $\boldsymbol{m}_{1}$ and $\boldsymbol{m}_{2}$ the endpoints of $M$. Recall that the feasible regions $G_{n}$ and $G_{m}$ are constructed by growing, respectively, the extended boundaries $M^{\prime}$ and $N^{\prime}$ by the distance $|\boldsymbol{m n}|+\delta$. Now suppose that $\boldsymbol{n}_{1}$ lies on the boundary of $G_{n}$ when $\delta=\delta_{n 1}$. From the construction of $G_{n}$, we must have $\delta_{n 1}+|\boldsymbol{m n}|$ equal to the shortest distance between $\boldsymbol{n}_{1}$ and the extended boundary $M^{\prime}$. At this point, if $\delta$ is still increased, $G_{n}$ will grow larger and contain a neighborhood of $\boldsymbol{n}_{1}$. Since $\boldsymbol{n}_{1}$ is an endpoint of $N$, its neighborhood must intersect $N_{x}$, which implies $\hat{F}_{n} \cap N_{x} \neq \emptyset$. This means that when considering endpoint $\boldsymbol{n}_{1}$ alone, it is necessary that $\delta$ can be increased only upto $\delta_{n 1}$ to ensure that $\hat{F}_{n} \cap N_{x}=\emptyset$. Therefore, by considering all four endpoints, we can thus compute the critical $\delta^{*}$ from the formula

$$
\begin{aligned}
\min & \left\{d_{s}\left(\boldsymbol{n}_{1}, M^{\prime}\right), d_{s}\left(\boldsymbol{n}_{2}, M^{\prime}\right),\right. \\
& \left.d_{s}\left(\boldsymbol{m}_{1}, N^{\prime}\right), d_{s}\left(\boldsymbol{m}_{2}, N^{\prime}\right)\right\}-|\boldsymbol{m n}|,
\end{aligned}
$$

where $d_{s}(\boldsymbol{p}, P)$ denotes the shortest distance between point $\boldsymbol{p}$ and boundary $P$. This, in turn, yields a capturing interval $\left[|\boldsymbol{m n}|,|\boldsymbol{m n}|+\delta^{*}\right]$.

An attractive property of this computed capturing interval is that when the distance between the fingers is in this interval, the object can be brought to the associated immobilizing configuration by moving the fingers toward each other until the distance between them is reduced to $|\boldsymbol{m n}|$. We can guarantee that the fingers will not be stuck anywhere else during this finger motion. This guarantee is clear from the fact that when finger $\mathcal{B}_{n}$ is in contact with the object, it can be in contact only with $N$ and, likewise, when finger $\mathcal{B}_{m}$ is in contact with the object, it can be in contact only with $M$. Because for all pairs of points, one from $M$ and the other from $N$, there exists only one equilibrium grasping configuration when one finger is at $\boldsymbol{m}$ and the other is at $\boldsymbol{n}$, it is then impossible for the fingers to get stuck anywhere except at the desired immobilizing configuration. 


\section{Preliminary Results}

We have implemented an algorithm for identifying all two-finger immobilizing grasps of type 1 and 2 for an input simple $n$-sided polygon. According to the condition given in Section II, our implementation straightforwardly verifies whether a grasp of type- 1 or type- 2 could be formed at each vertex/edge pair and vertex/vertex pair. For each grasp found, it then computes the corresponding capturing interval using the calculation provided in Section IV-B. It finally sorts all the grasps found according to the size of their capturing intervals. Checking the condition for type- 1 or type- 2 grasps takes constant time, so identifying the grasps takes $O\left(n^{2}\right)$. Since The number of concave vertices is in $O(n)$, the number of grasps found is in the order of $O\left(n^{2}\right)$. Sorting them takes $O\left(n^{2} \lg n\right)$, therefore we obtain an $O\left(n^{2} \lg n\right)$ implementation.

The program is written in $\mathrm{C}++$ and runs on an 800 $\mathrm{MHz}$ PC. It takes less than 0.1 second for any test case using a polygon with about 30 vertices. Fig. 8(a) and $8(\mathrm{~b})$, respectively, show the grasps of type- 1 and type-2 with the largest capturing interval. In the figures, immobilizing configurations are marked with circles and the largest distance where the two fingers can be far apart is represented by a line segment. We can see that the size of the capturing interval for the type-1 grasp in Fig. 8(a) is much larger than that of the capturing interval for the type-2 grasp in Fig. 8(b). For a grasp of type-2, when the fingers are expanded by small amount, the object is allowed to rotate by a relatively large angle. As shown in Fig. 8(c), to obtain a large capturing interval for type-2, the involved grasped edge has to be long enough to prevent large rotation from freeing the object from the capturing fingers. This somewhat suggests that a grasp of type- 1 is generally more robust than a grasp of type-2. In Fig. 9, type-1 grasps with the maximum capturing intervals are shown. For test polygons in Fig. 9(a) and Fig. 9(b), we found respectively 7 and 19 type- 1 grasps, and 6 and 26 type-2 grasps.

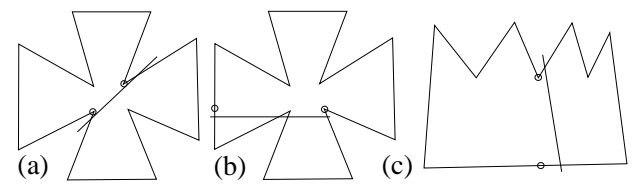

Fig. 8. Some results showing grasps with maximum capturing interval (a) type-1, (b) type-2 and (c) type-2 with a large grasped edge

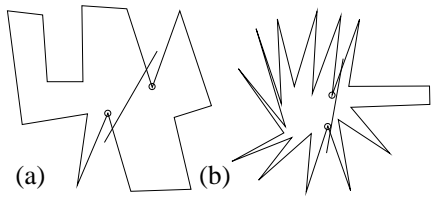

Fig. 9. More results showing type-1 grasps with the largest capturing interval

\section{Discussion AND CONCLUSION}

We have presented a classification of frictionless twofinger immobilizing grasps of polygons and a simple method for computing a capturing interval associated with a squeezing immobilizing grasp. With this computation, the separation distance between the fingers can be set during the pre-grasp stage to ensure that the object will never escape during the entire grasp execution and finally will be immobilized at the desired configuration.

The capturing interval computation presented in this paper takes into account only incident edges of concave vertices. We plan to consider more neighboring edges around the grasped concavities. This inclusion is likely to result in larger capturing intervals. Other future works include the investigation of issues concerning stretching immobilizing grasps and the computation of two-finger capturing intervals for $2 \mathrm{D}$ curved and $3 \mathrm{D}$ polyhedral and curved objects.

\section{REFERENCES}

[1] R. Berretty, K. Goldberg, M. H. Overmars, and A. Frank Van der Stappen. Orienting parts by inside-out pulling. In IEEE Int. Conf. on Robotics and Automation, 2001.

[2] A. Bicchi and V. Kumar. Robotic grasping and contact: A review. In IEEE Int. Conf. on Robotics and Automation, 2000.

[3] K. Gopalakrishnan and K. Goldberg. Gripping parts at concave vertices. In IEEE Int. Conf. on Robotics and Automation, 2002.

[4] E. Rimon and A. Blake. Caging 2D bodies by one-parameter twofingered gripping systems. In IEEE Int. Conf. on Robotics and Automation, pages 1458-1464, Minneapolis, MN, April 1996.

[5] E. Rimon and J. Burdick. New bounds on the number of frictionless fingers required to immobilize $2 \mathrm{~d}$ objects. In IEEE Int. Conf. on Robotics and Automation, 1995.

[6] A. Sudsang. A sufficient condition for capturing an object in the plane with disc-shaped robots. In IEEE Int. Conf. on Robotics and Automation, 2002.

[7] A. Sudsang and J. Ponce. A new approach to motion planning for disc-shaped robots manipulating a polygonal object in the plane. In IEEE Int. Conf. on Robotics and Automation, Sanfrancisco, CA, 2000.

[8] A. Sudsang, J. Ponce, and N. Srinivasa. Grasping and in-hand manipulation: Geometry and algorithms. Algorithmica, 26(3):466493, 2000.

\section{APPENDIX}

Proof of Lemma 1: Recall the condition of the equilibrium grasp of type 1 that the interior of the cone bounded by the lines of forces from one finger must cover the center of the other finger. Here, the left and right edges of the cone at $\boldsymbol{m}$ are drawn as $M_{L}$ and $M_{R}$. Note that we know that $M_{L}$ is perpendicular to $M_{1}$ and $M_{R}$ is perpendicular to $M_{2}$ by concavity of vertex $\boldsymbol{m}$ and by the fact that $M_{1}$ and $M_{2}$ are listed in counterclockwise order around the object (Fig. 4(a)). For the interior of the cone at $\boldsymbol{m}$ to cover the vertex $\boldsymbol{n}$, the segment $\overline{\boldsymbol{m} n}$ must be to the left of $M_{R}$ which implies that $M_{2}-\{\boldsymbol{m}\}$ cannot be in $K$. Similarly, this condition constrains that $\overline{\boldsymbol{m n}}$ must be to the right of $M_{L}$ which likewise implies that $M_{1}-\{\boldsymbol{m}\}$ can neither be in $K$. Applying the same argument to the cone at $\boldsymbol{n}$, we conclude that all the boundary segments $M_{1}, M_{2}, N_{1}$ and $N_{2}$ are not in $K$ except at the endpoints $\boldsymbol{m}$ and $\boldsymbol{n}$. 